\title{
Efficient FEM-FBM for Simulation of Elliptic Particle Sedimentation with Thermal Convection
}

\author{
Antariksha Verma, H. K. Suhas
}

\begin{abstract}
The dynamic sedimentation of large particles including thermal convection gained the significant attentions in various applications using the Direct Numeral Solution (DNS) methods. The current solutions are mainly focused on isothermal suspended particles without the thermal convection separating dissolved particles and enveloping fluids. The systems beside thermal convection having the lack of sufficient investigations like missing the hot and cold elliptic particle in infinity long channels. In this work, we work on two challenges efficient DSN method designing and simulation of elliptic particle in infinity long channel using proposed DNS. The novel approach presented to study and simulates dynamic behaviour of elliptic particle sedimentation using the different settling modes using the Swarm Intelligent (SI) construct Finite Element Fictitious Boundary Method (FEM-FBM) and validates outcomes effectively. The Particle Swarm Optimization (PSO) used as SI to improve the accuracy and efficiency of FEM-FBM model in this work. The PSO based multi-grid FEM-FBM called PFEM-FBM proposed as Direct Numerical Solution (DNS) to simulate and validate fluid flows with thermal convection. The PFEM-FBM method used to understand the progression, energy, and warmth conditions effectively. The re-enactments utilizing PFEM-FBM led to utilizing the three diverse experiments. In the principal case, the relative investigation of cold, hot, and isothermal elliptic particles with thermal convection introduced. In the resulting situation, the estimation of cold elliptic particle improving in an incomprehensibly long channel with its endorsement presented. In the third case, the examination of hot elliptic particle resolve in an incessantly large channel with its support presented. The completion of the study is validated using the simulation outcomes with recent technique at the end.
\end{abstract}

Keywords: Direct numerical solution, finite element method, fictitious boundary method, particle swarm optimization, sedimentation, thermal convection.

\section{INTRODUCTION}

The action of strong particle in a gooey liquid has big numbers of applications into the deferent planning areas. For instance, into the re-dox of suspension stream batteries, shape, size \& course of action of all powerful elements of material \& material conductive particles are typically combined parameters impacting theology \& transport

Revised Manuscript Received on February 06, 2020.

* Correspondence Author

Antariksha Verma* PhD Scholar, Department of Mechanical Engineering, Kalinga University, Raipur, India.

Dr. H. K. Suhas, Department of Mechanical Engineering, Kalinga University, Raipur, India.

(C) The Authors. Published by Blue Eyes Intelligence Engineering and Sciences Publication (BEIESP). This is an open access article under the CC BY-NC-ND license (http://creativecommons.org/licenses/by-nc-nd/4.0/) characteristics of suspension liquid. Into such programmes, the liquid inertness \& thickness has been restricted, director of liquids \& solid particles has been unequivocally combined, and thusly, molecule movement demonstrates rich physical wonders [1]. Among various parameters impacting molecule movement, molecule shapes expect a fundamental activity. During the previous quite a few years, the motion of circular particles has attracted much consideration because of the balance of particle shape. As of late, more inquiries about the spotlight in movement of non-round particles to really discover genuine molecule transport structures [2]. For two dimensional (2D) bended molecule or three dimensional (3D) ellipsoid molecule sedimentation, 8 undeniable conditions has been represented: vertical mode, oscillatory mode, flat mode, inclined mode, level II mode, inclined II mode, odd moving mode \& winding model. Even mode suggests molecule sediment on a level plane with an enduring speed with centre line of the channels [3] [4]; when flat II mode implies molecule build-up on a level plane with the faltering model. In the accepted style, particle build-up along predictable speed and an unfaltering propensity to level; while in the inclined II mode, inclined molecule leftovers with the influencing structure. Into vertical mode, molecule residue vertically \& it will in general seen such a restriction of inclined mode along a propensity purpose ninety degrees. Oscillatory mode suggests molecule squirms fall channel, pushing toward various areas of divider at times \& influencing in channel centre line; when atypical moving mode defined as falling elements turns as if it has been coming to \& moving up with one of the channel dividers. Winding mode has been surprising into the 3D ellipsoid molecule settlement \& it exhibits iota spirals within channel centreline when edge between molecule centre point \& channel centreline keeping predictable [5]. As of late referenced modes all things considered result from 2 impacts: 1 st is waterway geometry impacts, which has been delineated through stalemate degree such channel width through molecule turn length; 2nd is molecule inertial impact, which has been depicted through molecule Reynolds number like segment molecule size \& molecule thickness. By a wide margin the greater part of the assessments talked about above concentrated on just iso-thermal alimented elements where there is no warm convection into alimented elements \& consolidating liquids [6] [7]. As warm impacts are not constantly irrelevant in pondering molecule suspension since warmth move may essentially change the particle kinematics, some ongoing works answered to think about the elliptic particle sedimentation with the thermal convection, be that as it may, there is absent of broad investigation of energy trade with thermal consequences for the sedimentation conduct of curved particles Different Settling Modes [8]-[10]. 


\section{Efficient FEM-FBM for Simulation of Elliptic Particle Sedimentation with Thermal Convection}

Hence with such motivation, in this research we model, simulate, and validate the sedimentation behaviour of elliptical particles with convention heat transfer using various settling modes.

In this paper we present the study that investigates the design of dynamic behaviour of elliptic particle sedimentation using the different settling modes using the PSO based multi-network Limited Component Invented Limit (PFEM-FBM) strategy. As far as we could possibly know this the first DNS approach in which the SI acquainted with streamline the multigrain issue solver. We planned, mimicked and approved the impacts of warm properties of the liquid, for example, warm enlargement and warm dissipating on the sedimentation of the hot and cold elliptic molecule in limitlessness long channel. In segment II, the short audit of related works exhibited. In area III, proposed strategy and calculations depicted. In segment IV, re-enactment results introduced. In area $\mathrm{V}$, the approval displayed. In segment VI, conclusion and future work described.

\section{RELATED WORK}

This section presents the various DNS methods introduced for the particular flows simulation followed by the various methods for sedimentation particle analysis presented.

In [11]-[14], author presented a multi-grid limited component invented limit strategy (FBM) to increase particular streams. Later past, various creamer methodologies is made to re-enact sedimentation of released particles in a liquid, As instance, the cross-zone Boltzmann framework (LBM) is gotten together with other numerical methodology, for example, IBM [15], Limited Component Strategy (FEM) [16] and Discrete Component Strategy (DEM) [17].

In [18] and [19], the creators utilized another cross breed technique CFD \& DEM, where calculated fluid components (CFD) has been associated along DEM for analysis of the sediment transportation. Despite the fact that heat move in particulate flows is engaged with numerous modern applications, yet just a couple of results are found in the writing. In [20], the creator proposed an Inundated Limit Technique (IBM) with regards to a Limited Volume (FV) set system plan for the plan of condition move in multiple two-dimensional geometries externally concerning the stable platform movement.

In [21], author displayed the FV-IB system with a non-deadened cross-section methodology for warmth move and liquid stream around fixed particles. Before [22], the creator utilized a compelled volume non-adjusted purpose of control fitted matrices to consider the warm convection around the fixed strong stage. In [23] [24], the writer utilized the Blend imagine for re-enactments of two dimensional molecule sedimentation issues along warmth move among elements \& joining liquid.

In [25], the writer used the imagined space procedure to deal with particulate stream issues and loosened up it for analyse heat convection in interface between liquid \& strong elements. In [26] [27] writer built up a compelled volume IBM framework for warmth move between liquid \& going strong elements in molecule stacked streams. The numerical assessment warm convection in particulate streams is endeavoured through specific various experts too in [28] [29].

In [30] author recognized five contending systems for horizontal relocation of a roundabout particle with thermal convection, in particular, the divider aversion because of grease, the inertial lift identified with sheer slip, the lift on account of particle insurgency, the lift in light of the twist of the regular velocity form also, the journey influenced by thermal convection. Some different scientists have additionally given an account of the numerical investigation of thermal convective particulate flows [31]-[39].

In [40], productive methodology for the precise reproduction of thermal convective particulate flows including countless particles exhibited. Some different scientists have likewise given an account of the numerical investigation of thermal convective particulate flows.

In [41], the writer revealed the main examination wherein the thermal consequences during the sedimentation head of extensive bits by means for particle resolved direct numerical multiplication including created some new approaches for particle's settling.

In [42], contributors analysed results convection of thermal on segments of an elliptic element when improving into impenetrable liquor utilizing multi-network FEM-FBM. The displayed system settles the warmth, force, and coherence conditions all the while.

In this paper, we motivated from the works reported in [40] and [42] to design the PSO construct multigrain FEM-FBM design to recreate the particulate streams with thermal convection.

\section{METHODOLOGY}

The swarm intelligent technique PSO introduced along with multi-grid FEM-FBM model to form the novel DNS technique called PFEM-FBM in this paper. The proposed model at that point used to re-enact and dissect the thermal convection impacts on the elliptic particle elements while improving in an impenetrable liquid.

\subsection{Problem Formulation}

To build the problems, we consider the list of parameters mentioned in the table 1 . It shows the $N$ solid particles of mass Mi where $\mathrm{i}=1,2 \ldots \mathrm{N}$ in impenetrable Newtonian vapour.

Table 1: List of parameters in fluid flow

\begin{tabular}{|l|l|}
\hline Symbol & Significance \\
\hline$N$ & solid particles number \\
\hline$M i$ & $N$ number of mass \\
\hline$p_{f}$ & Fluid density \\
\hline$V$ & Fluid viscosity \\
\hline$\Omega_{f}(t)$ & Domain accepted through fluid at time $t$ \\
\hline$\Omega_{i}(t)$ & $\begin{array}{l}\text { Domain accepted through fluid of } \\
i^{\text {th }} \text { particle at time } t\end{array}$ \\
\hline$\Omega_{T}$ & Total domain \\
\hline $\mathrm{U}$ & Fluid velocity \\
\hline$\mu_{f}$ & Coefficient of viscosity \\
\hline
\end{tabular}




\begin{tabular}{|l|l|}
\hline$F$ & Source term \\
\hline$T$ & Temperature \\
\hline \multicolumn{1}{|l|}{$c_{f}$} & Specific heat \\
\hline$k_{f}$ & Thermal conductivity of fluid \\
\hline$\sigma$ & Fluid phase total stress tensor \\
\hline
\end{tabular}

The $\Omega_{T}$ as total domain has been shown by:

$$
\Omega_{T}=\Omega_{f}(t) \cup \Omega_{i}(t) \quad \forall i \in(1,2,3, \ldots N)
$$

(1)

where,

- $\Omega_{T}$ Is an every calculation empire individualistic of $\mathrm{t}$ ?

- $\Omega_{f} \& \Omega_{i}$ be conditional on the time $t$, hence it is denote as $\Omega_{f}(t)=\Omega_{f}$ and $\Omega_{i}(t)=$ $\Omega_{i}$ release $\mathrm{t}$ in the notations.

- $\partial \Omega_{i}$ Appear for the margins of the $i^{\text {th }}$ particle.

Based on this, the fluid flow model and particle motion model are formulated as:

\section{A. Model of Fluid Flow}

The incompressible fluid motion with $p_{f}$ is administered through the conditions of force, vitality, and coherence in the space $\Omega_{f}$ given by:

$$
\begin{aligned}
& \nabla . u=0 \quad(a) \\
& p_{f}\left[\frac{\partial u}{\partial t}+u \cdot \nabla u\right]-\nabla \cdot \sigma=\mathrm{f}(\mathrm{b}) \\
& p_{f} c_{f}\left[\frac{\partial u}{\partial t}+u \cdot \nabla T\right]=k_{f} \nabla^{2} T
\end{aligned}
$$

(4)

Where, $\sigma$ which is given by:

$$
\sigma=-p I+\mu_{f}\left[\nabla \mu+\left(\nabla \mu^{\mathrm{T}}\right)\right]
$$

\section{B. Model of Particle Motion}

After defining the model of fluid flow, this section presents the model of particle motion. As the inflexible particles free, they move in the fluid space which prompts the way that particles furnished with a rotational and translational motion under the activity of gravity and powers, for example, impact powers and hydrodynamic powers. The hydrodynamic powers brought about by fluid and crash powers brought about by associations among particle-divider or particle-particle. To assemble the model the rundown of parameters is given in table 2 .

Table 2: List of parameters in particle motion

\begin{tabular}{|c|l|}
\hline $\begin{array}{c}\text { Sym } \\
\text { bol }\end{array}$ & Significance \\
\hline$U_{i}$ & $i^{\text {th }}$ particle Translational velocity \\
\hline$w_{i}$ & $i^{\text {th }}$ particle Angular velocity \\
\hline$m_{i}$ & Mass of $i^{\text {th }}$ particle \\
\hline$m_{f}$ & Fluid mass accepted similar volume as $m_{i}$ \\
\hline$F_{i}$ & $\begin{array}{c}\text { Resultant hydrodynamic (lift } \& \text { drag coefficients) of } i^{t h} \\
\text { particle }\end{array}$ \\
\hline$F_{i}^{\prime}$ & Collision forces action on of $i^{\text {th }}$ particle \\
\hline$I_{i}$ & $i^{\text {th }}$ particle inertia tensor interaction \\
\hline$\tau_{i}$ & Resultant torque centre of mass of $i^{\text {th }}$ particle \\
\hline $\mathrm{g}$ & Gravitational accelerations \\
\hline
\end{tabular}

The solid particles motion conducted by Newton-Euler formulations, accordingly the particle required to satisfy the equation (6) if the translational and rakish speeds of particle available:

$$
\begin{aligned}
& m_{i} \frac{d U_{i}}{d t}=\left(\Delta m_{i}\right) g+F_{i}+F_{i}^{\prime}, \quad d_{i} \frac{d w_{i}}{d t}+w_{i} \times \\
& \left(I_{i} w_{i}\right)=\tau_{i} \\
& \Delta \mathrm{m}_{i} \text { Is given by: } \\
& \Delta m_{i}=m_{i}-m_{f}
\end{aligned}
$$

\section{Effects of Fluid Interactions}

The particles are free and they move which leads the interactions among the particles and fluid. The effects of such interactions resulted into exertion of the forces on fluid and particles. The trading of vitality and force happens among fluid and particles during the interactions such as hydrodynamic forces, momentum interaction, energy interaction, and Bossiness approximation. The equations of such problems described in [40] [42]. To solve such problems related to interactions the solver model introduced in next section.

\subsection{Multi-grid PFEM-FEB}

To solve the fluid particle interactions we introduced the multi-grid approach to solve which depends in various networks, gotten by routinely refining the course work? Essentially multi-lattice used to get the best answers to tackle the issue, in this work we utilized the PSO technique in the multi-network solver. In multi-matrix, the PSO imperative is applied to the lingering in the wake of softening on all activity levels, and a straightforward moving through solver is applied to make the course of action in coarsest grid if amount of degrees chance is close to nothing. Further, prolongation has been utilized trailed through post smoothing in PSO health ability to get the best particle plan. These schemes remain continuously the accentuations of multi cross section cycle (V \& F cycle, as appearing in calculation 1 and 2) completed as a major aspect of the PSO intermingling step. With the assistance of certain administrators, here we clarified how multi-framework deals with an issue utilizing distinctive network levels. The multi-lattice method to comprehend the straight framework $A_{i} u_{i}=b_{i}$ is presented in the following steps with the $k$ number iterations. Figure 1 demonstrates the PSO based multi-grid solver introduced in this work. Using the iterative optimization over the various grid levels, better results obtained to solve the problems. Based on the figure 1, the algorithm 1 and 2 designed to solve the problems caused by the fluid particle interactions. The multi-framework $\mathrm{V}$ cycle (MGV), multi network W cycle (MGW) \& multi matrix F cycle (MGF) required by using proposed DNS method. The algorithm 1 and 2 shows the steps solving process of MGV and MGF respectively. 


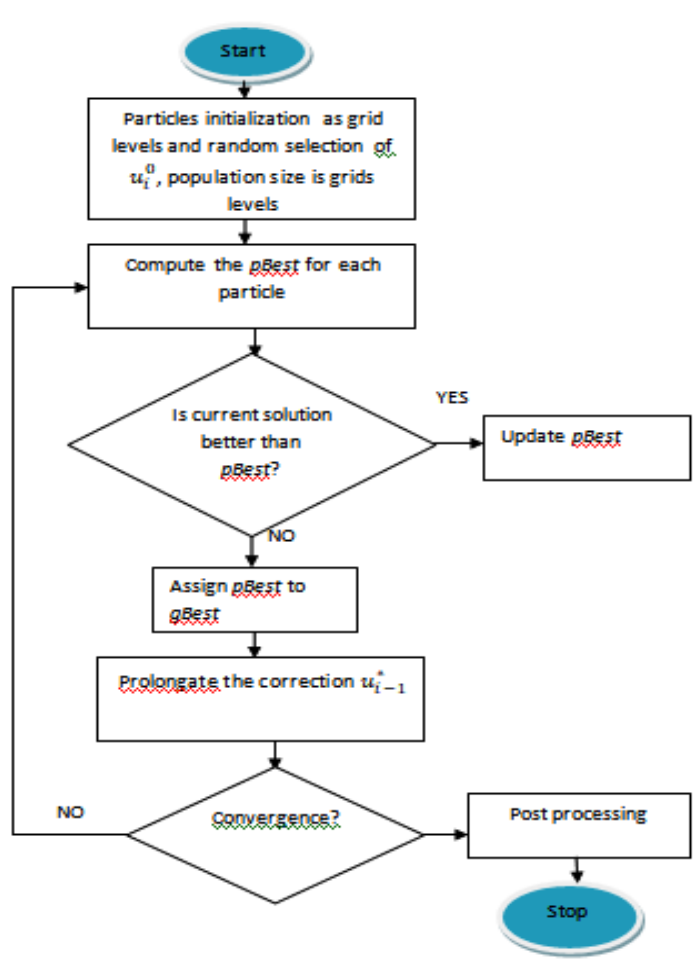

Fig.1. PSO based multi-grid solver

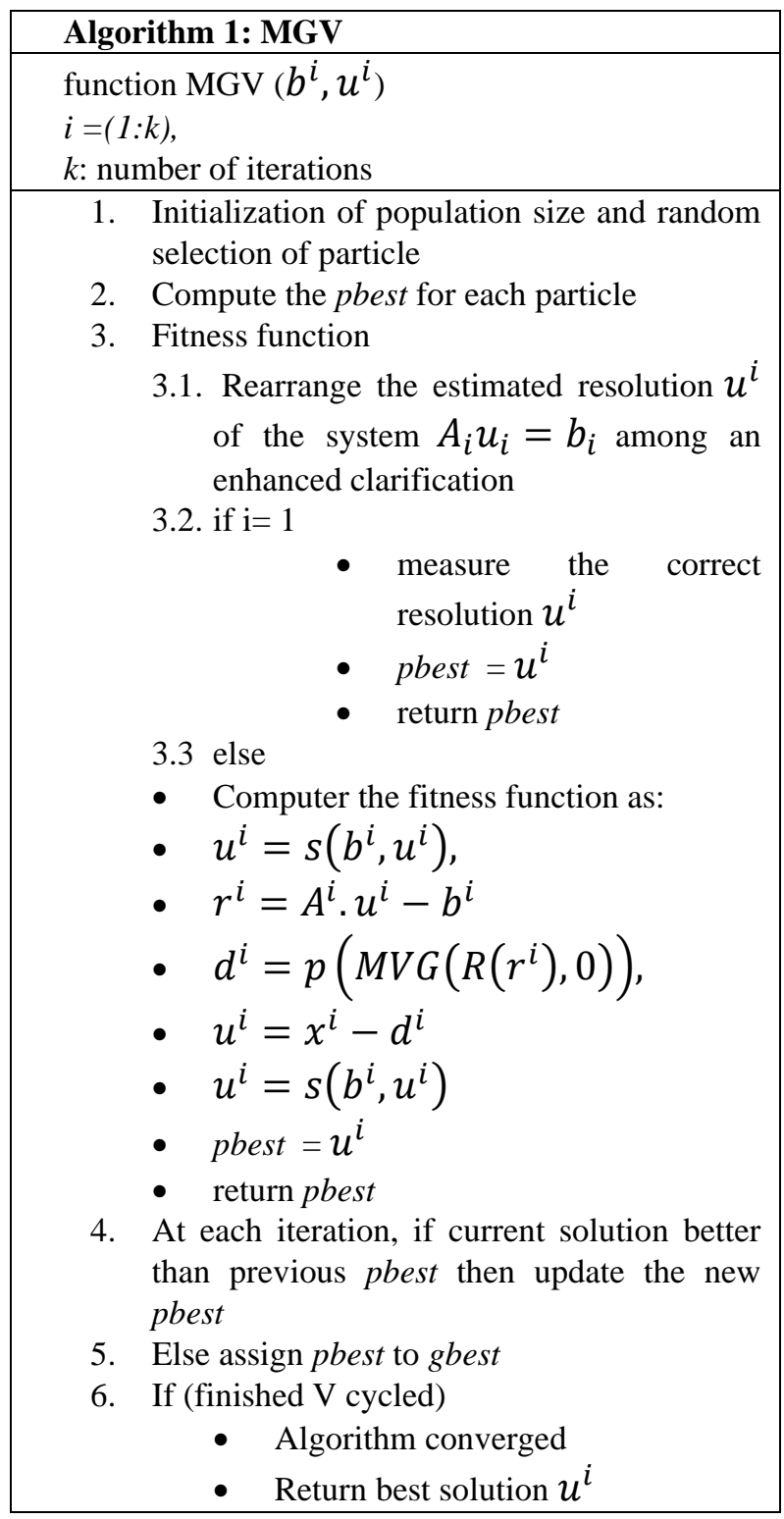

7. Apply post process on obtained $u^{i}$

8. Return final $u^{i}$

\begin{tabular}{|l}
\hline \multicolumn{2}{|l|}{ Algorithm 2: MGF } \\
\hline function MGF $\left(b^{i}, u^{i}\right)$ \\
\hline 1. Return an accurate solution $u^{i}$ of the system \\
\\
$A_{i} u_{i}=b_{i}$ \\
2. solve exactly $A_{i} u_{i}=b_{i}$ to get $u^{1}$ \\
3. for $\mathrm{i}=2$ to $\mathrm{k}$ \\
4. $u^{i}=M G V\left(b^{i}, p\left(u^{i}\right)\right)$ \\
5. end for
\end{tabular}

After presenting the PSO based multi-grid solver, next we have to present the model of Multi-grid PFEM-FBM solver. This multi-matrix invented limit technique depends on the PFEM foundation framework which covers the entirety computational domain $\Omega_{T}$ as described in [40] [42]. Using the solver model, we further perform the computations to solve the problems related to hydrodynamic forces, torque, drag and lift coefficients, energy balance equations, dimensionless parameters which are used to investigate the dynamic sedimentation behaviour of particle with thermal [42].

\section{SIMULATION RESULTS}

The simulation and validation of PFEM-FBM approach performed using the MATLAB tool in this paper by considering the three test cases described as below:

\subsection{Investigation of Different Elliptical Particle}

\section{Sedimentation}

In this test case, settling practices of a the hot elliptic molecule, the whole distance open to the fierce segments liquid, a cool molecule in the hot liquid, and an isothermal molecule in a vertical liquid channel are re-enacted using the diversion parameters depicted in the table 3 . The article is from the start arranged at the point $(2,10)$, and its course is with the ultimate objective that the genuine centre of oval creates the edge of $\theta=0^{\wedge}$ Oto the horizontal centre point of channel. For host and cold particle, the distinct the dimension less temper of particle \& fluid has been 1 . The differentiation in temperatures is 0 for the isothermal particle.

Table 3: Simulation parameters in case study 1

\begin{tabular}{|c|c|c|}
\hline Parameter & lue $^{\text {Va }}$ & Significance \\
\hline $\mathrm{b}$ & $5^{0 .}$ & Ellipse minor axis \\
\hline A & $2 b$ & Major axis of ellipse \\
\hline $\mathrm{W}$ & 4a & Width of channel \\
\hline $\mathrm{H}$ & 12 & Height of channel \\
\hline $\mathrm{L}$ & $\sqrt{a b}$ & $\begin{array}{c}\text { elliptic particle length of } \\
\text { characteristic }\end{array}$ \\
\hline
\end{tabular}




\begin{tabular}{|c|c|c|}
\hline $\mathrm{V}$ & $1^{0 .}$ & Fluid kinetic viscosity \\
\hline$p_{r}$ & $1^{1 .}$ & density ratio of solid fluid \\
\hline $\operatorname{Re}$ & 40 & Dimension less flow number values \\
\hline $\operatorname{Pr}$ & $7^{0 .}$ & Prandtl number \\
\hline$c_{r}$ & 1 & solid \& liquid Heat power ratio \\
\hline $\mathrm{T}$ & 1 & $\begin{array}{l}\text { particle surface dimension less } \\
\text { temperature }\end{array}$ \\
\hline$T_{f}$ & 0 & Fluid temperature \\
\hline$G_{r}(h o t)$ & $0^{50}$ & Grashof number for hot \\
\hline$G_{r}($ cold $)$ & $00^{-5}$ & Grashof number for cold \\
\hline$G_{r}$ (isothermal) & 0 & Grashof number for isothermal \\
\hline
\end{tabular}

Figure 2 shows the cold particle similarly increases a lot big possible velocity than hot \& isothermal particle \& therefore, there is an immense variation between drag coefficients Album of hot moreover cold elliptic particles when spot achieve most extreme speeds like appeared in figure 3 . From these outcomes of cold particle, it is seen that the trademark convection improves the compelled convection, and thusly, the settling speed of the molecule is incredibly high. The eventual outcomes of hot molecule settling in a chilly liquid exhibit that molecule goes about as a glowing source and the glow streams from the molecule for including liquid. Both drag control \& delicacy power shield molecule via reviving at a higher space toward gravity, \& molecule settles influenced by compete between warm convection \& compelled convection. Appropriately, settled speed of hot molecule in chilly liquid low appears differently in relation to the cool molecule into hot liquid and that of iso-thermal molecule.

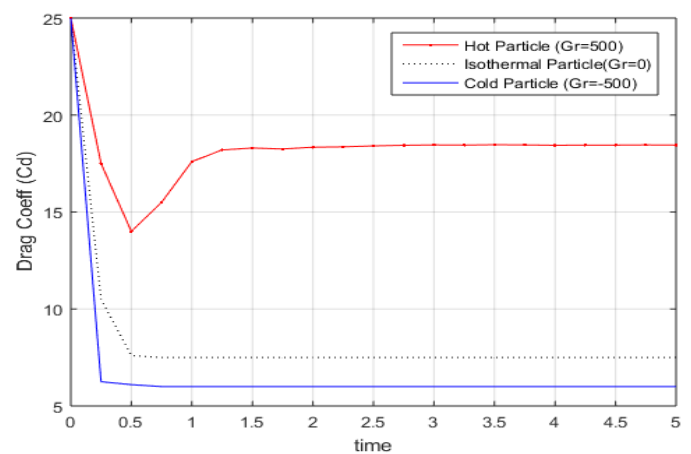

Fig.2. Drag coefficient of the particles with respect to time

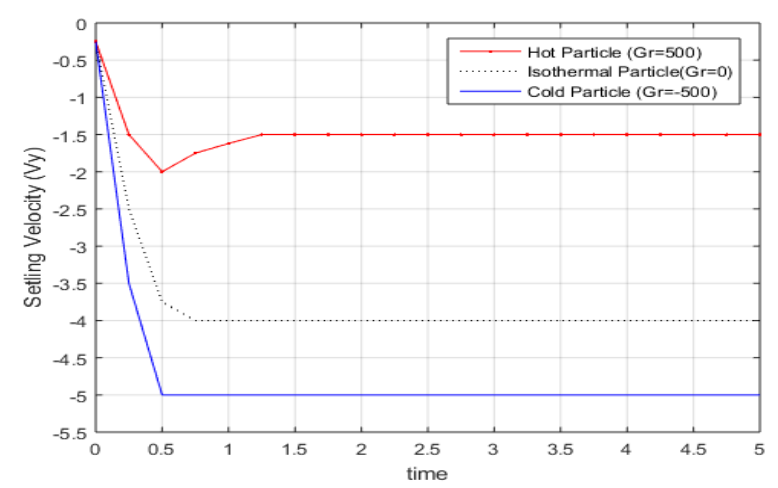

Fig.3. Settling velocity of the particles with respect to time

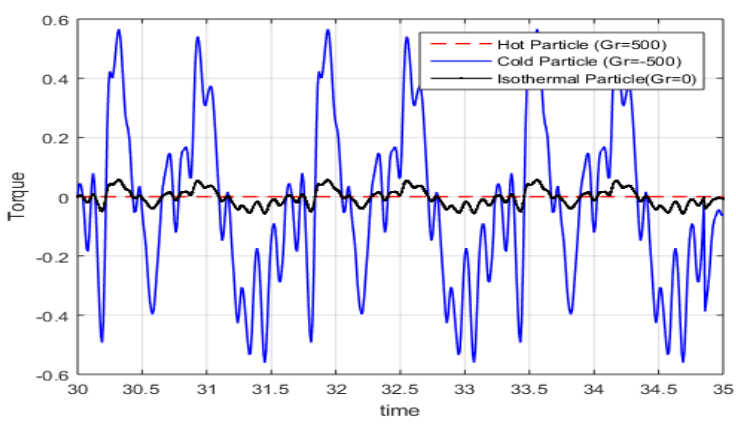

Fig.3. Torque of particles with respect to time

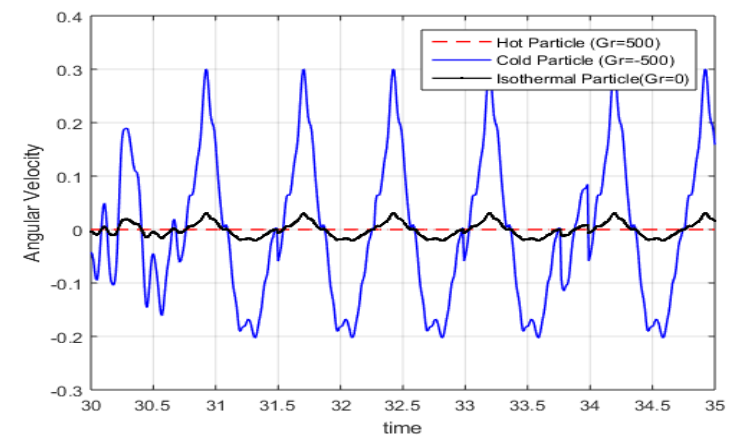

Fig.5. particles Angular velocity along respect to time

The isothermal particle sedimentation results show that all fluid \& particle are at a comparable steady heat; along these lines, power in light of thermal convection is excluded. The particle settles simply influenced by obliged convection in light of the way that no glow change happens among the liquid and molecule that makes the sophistication control. Further, in figures 4 and 5, it is seen that in light of big settled speed of the chilly molecule, plentifulness of torque powers following up on molecule as a result of hydrodynamic investment is higher showed up distinctively in connection to the hot and isothermal particles. Similarly, the isothermal molecule faces a torque of little monstrosity, in any case, the level of torque powers following up on the hot molecule is just around zero because of more slow settling velocity.

\subsection{Investigation of Cold Elliptical Particle Settling}

In this segment, we further exhibited the re-enactment after-effects of the third experiment where the examination of cold curved particle settling in hot fluid performed under the unmistakable settling strategies for a roundabout particle with the impact of compelled and thermal convection. Table 4 exhibits the once-over of re-enactment parameters for this test. The elliptic particle has been added at centreline of channel with the intention which has been outstanding centre of particle creates edge of $\theta=\llbracket 45 \rrbracket^{\wedge} \mathrm{O}$ to level centre of the flow.

Table 4: Simulation parameters in case study 2

\begin{tabular}{|l|l|l|}
\hline Parameter & Value & Significance \\
\hline $\mathrm{b}$ & 0.5 & ellipse small axis \\
\hline $\mathrm{a}$ & $2 \mathrm{~b}$ & Major axis of ellipse \\
\hline $\mathrm{W}$ & 0.4 & Width of channel \\
\hline $\mathrm{H}$ & 2 & Height of channel \\
\hline $\mathrm{L}$ & $\sqrt{a b}$ & $\begin{array}{c}\text { elliptic particle } \\
\text { Characteristic length }\end{array}$ \\
\hline
\end{tabular}


Efficient FEM-FBM for Simulation of Elliptic Particle Sedimentation with Thermal Convection

\begin{tabular}{|c|c|c|}
\hline $\mathrm{v}$ & 0.1 & $\begin{array}{l}\text { kinematic viscosity of } \\
\text { the fluid }\end{array}$ \\
\hline$p_{r}$ & 1.001 & $\begin{array}{l}\text { fluid-solid density } \\
\text { ratio }\end{array}$ \\
\hline $\operatorname{Re}$ & 100 & $\begin{array}{c}\text { dimensionless } \\
\text { number values }\end{array}$ \\
\hline $\operatorname{Pr}$ & 7 & Prandtl number \\
\hline$c_{r}$ & 1 & $\begin{array}{l}\text { solid and liquid Heat } \\
\text { capacity ratio }\end{array}$ \\
\hline $\mathrm{T}$ & 0 & $\begin{array}{l}\text { dimensionless } \\
\text { temperature }\end{array}$ \\
\hline$T_{f}$ & 1 & Fluid temperature \\
\hline$G_{r}$ & $\begin{array}{l}\{-200,-600, \\
-1000\}\end{array}$ & 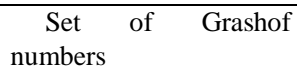 \\
\hline
\end{tabular}

particle influences, as rolling mode particle demonstrates steady rotational movement.
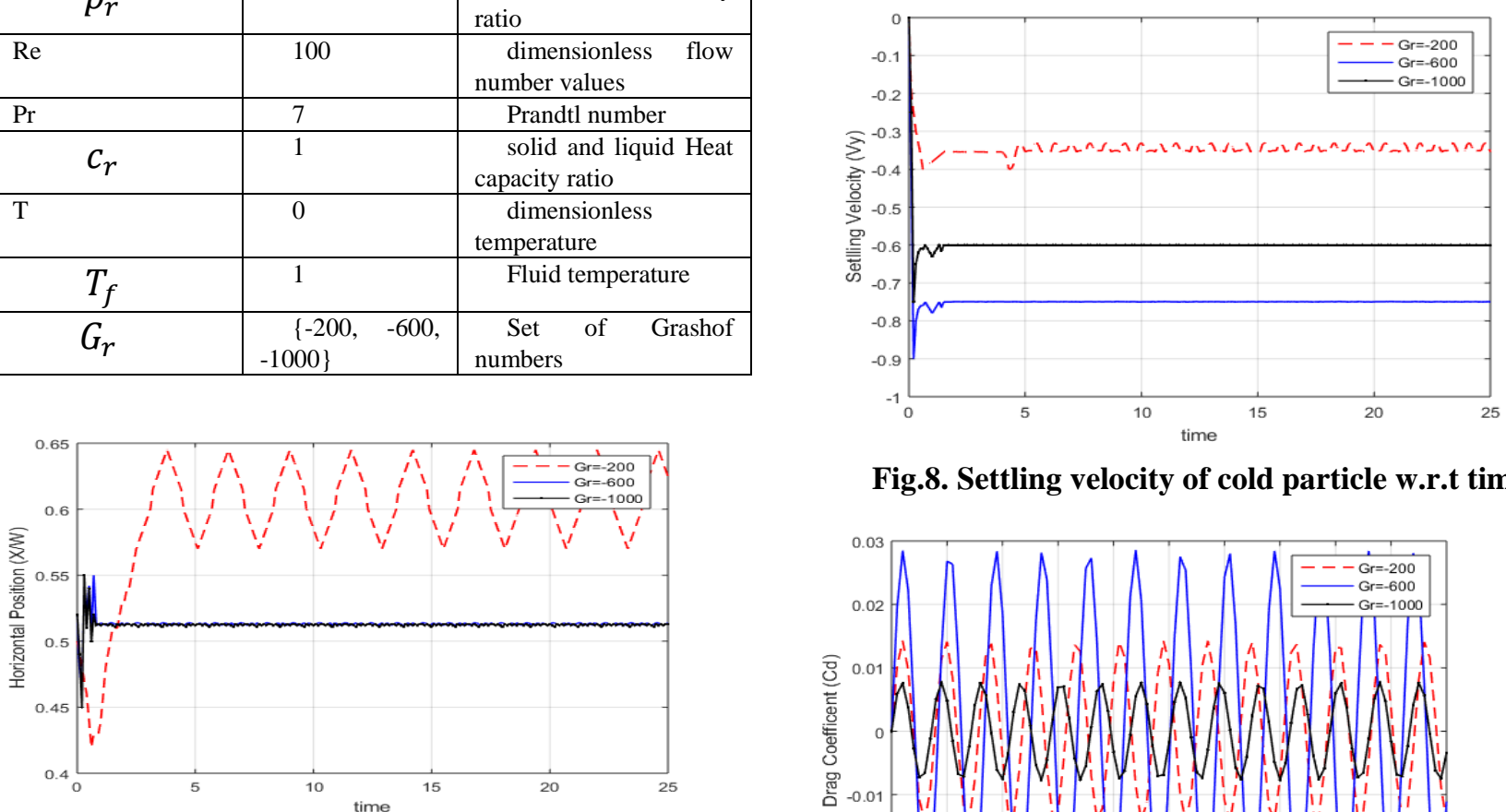

Fig.6. Horizontal position of settling cold particle w.r.t time

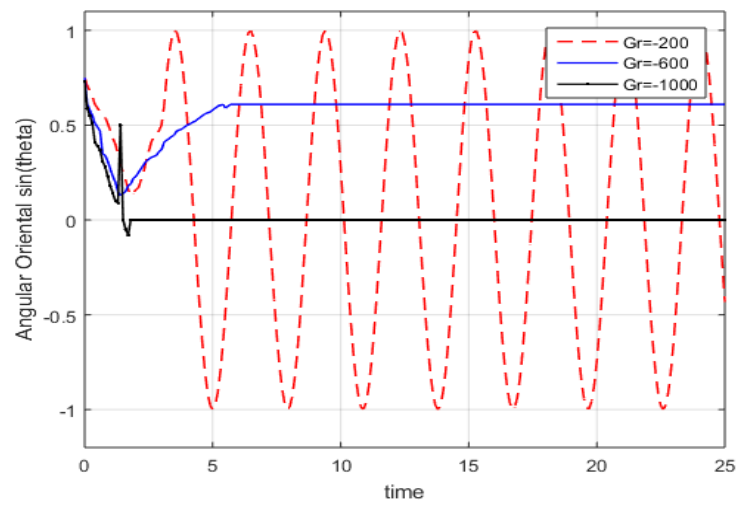

Fig.7. cold particle w.r.t time Angular orientation of settling

Figures 6 and 7 are showing the results of three unmistakable sedimentation methods of the cold elliptic particle with changing Gr numbers. For the instance of $\mathrm{Gr}=$ 200, it is seen that the settled elliptic particle shows the tumbling method. For occurrence of $\mathrm{Gr}=-600$ particle improves continually in inclined method close centreline of pipe along reliable list to regular accomplishment of flow. For the case of $\mathrm{Gr}=-1000$, the elliptic particle settled frequent with centreline in horizontal mode with a predictable purpose of $\theta=0^{\wedge} \mathrm{o}$ to the horizontal rotate. The modes change to ensure that the effect of ordinary thermal convection over particle sedimentation increases as the Grashof number augmentations.

Figure 8 shows that particle settling velocity in the tumbling mode moreover demonstrates standard irregular motions with a little abundance, while particles covered along enduring velocity in tending mode $\&$ horizontal mode. From this result, it is perceived that descending velocity has been big in inclined mode appeared differently concerning horizontal mode, in like manner, settled velocity of rolling

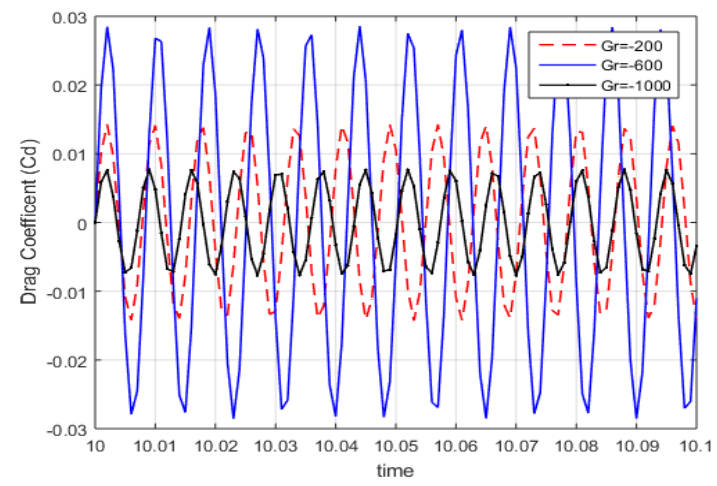

Fig.9. Drag coefficient of cold particle w.r.t time

Figure 9 demonstrates the outcome of drag coefficient following up on the particle in various modes, for example, tumbling mode, slanted mode, and even mode. These outcomes guarantee that the ampleness of the drag coefficient in the slanted mode is higher stood apart from the other two modes by righteousness of the higher settling speed. Drag coefficient plentifulness in the tumbling mode is higher than that in the flat mode positioning in light of a higher recurrence of sideways motions and precise pivots.

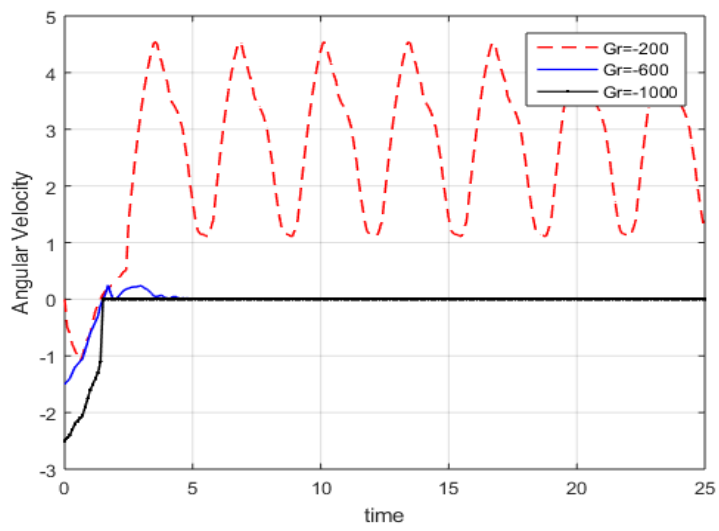

Fig.10. Angular velocity acting on settling cold particle w.r.t time

Finally in investigation of cold elliptic particle settling, the angular velocity and torque measured in figures 10 and 11 respectively. The results show that greatness of torque is practically steady zero on account of flat mode and slanted mode positioning,

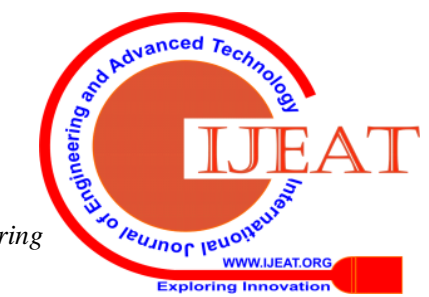


and thus the rotational speed of the molecule in these two modes is zero. As appearing in figure 10, the cool elliptic molecule in the tumbling mode settling encounters a solid coupled power the counter-clockwise way. Figure 11 exhibits that jaunty expanding pace created \& particle turns with exact velocity whose significance changes sometimes along every turn of particle.

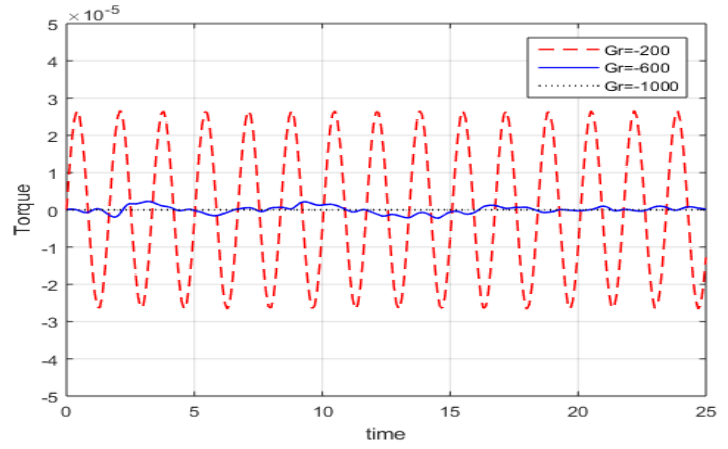

Fig.11. Torque acting on settling cold particle w.r.t time

\subsection{Investigation of Hot Elliptical Particle Settling}

The final investigation of this research work presented in this section in which analysis of hot elliptic particle sedimentation exposed to the harsh elements fluid considered. The re-enactment parameters are exhibited in table 5. As talked about before the sedimentation of hot elliptic particle is investigated in three modes such as vertical, rolling, and horizontal. In subsections we analyze the simulation results in each mode using the PFEM method.

Table 5: Simulation parameters in case study 3

\begin{tabular}{|c|c|c|}
\hline Parameter & Value & Significance \\
\hline $\mathrm{b}$ & 0.5 & Minor axis of ellipse \\
\hline $\mathrm{a}$ & $2 \mathrm{~b}$ & Major axis of ellipse \\
\hline $\mathrm{W}$ & 0.4 & Width of channel \\
\hline $\mathrm{H}$ & 2 & Height of channel \\
\hline $\mathrm{L}$ & $\sqrt{a b}$ & $\begin{array}{l}\text { Characteristic length of } \\
\text { the elliptic particle }\end{array}$ \\
\hline $\mathrm{v}$ & 0.1 & $\begin{array}{l}\text { kinematic viscosity of } \\
\text { the fluid }\end{array}$ \\
\hline$p_{r}$ & 1.001 & $\begin{array}{l}\text { fluid-solid density ratio } \\
\text { (vertical position and } \\
\text { velocity analysis) }\end{array}$ \\
\hline$p_{r}$ & 1.04 & $\begin{array}{l}\text { fluid-solid density ratio } \\
\text { in vertical mode settling }\end{array}$ \\
\hline$p_{r}$ & 1.07 & $\begin{array}{l}\text { fluid-solid density ratio } \\
\text { in rolling mode settling }\end{array}$ \\
\hline$p_{r}$ & 1.7 & $\begin{array}{l}\text { fluid-solid density ratio } \\
\text { in horizontal mode settling }\end{array}$ \\
\hline $\operatorname{Re}$ & 100 & $\begin{array}{l}\text { dimensionless flow } \\
\text { number values }\end{array}$ \\
\hline $\operatorname{Pr}$ & 7 & Prandtl number \\
\hline$c_{r}$ & 1 & $\begin{array}{l}\text { solid liquid Heat } \\
\text { capacity ratio }\end{array}$ \\
\hline $\mathrm{T}$ & 1 & \begin{tabular}{ll}
\multicolumn{1}{c}{ particle surface } \\
dimensionless \\
temperature
\end{tabular} \\
\hline$T_{f}$ & 0 & Fluid temperature \\
\hline$G_{r}$ & 200 & $\begin{array}{l}\text { Grashof number } \\
\text { (vertical position and } \\
\text { velocity analysis) }\end{array}$ \\
\hline$G_{r}$ & 400 & $\begin{array}{ccc}\text { Grashof number in } \\
\text { vertical mode settling }\end{array}$ \\
\hline$G_{r}$ & 600 & $\begin{array}{l}\text { Grashof number in } \\
\text { rolling and horizontal } \\
\text { mode settling }\end{array}$ \\
\hline
\end{tabular}

A. Vertical Mode

In vertical mode, examinations performed utilizing the estimation of $\mathrm{pr}=1.04$ and $\mathrm{Gr}=400$ with every other parameter comparative as delineated in Table 5. The particle settles affected by the challenge between the regular convection and the constrained convection.

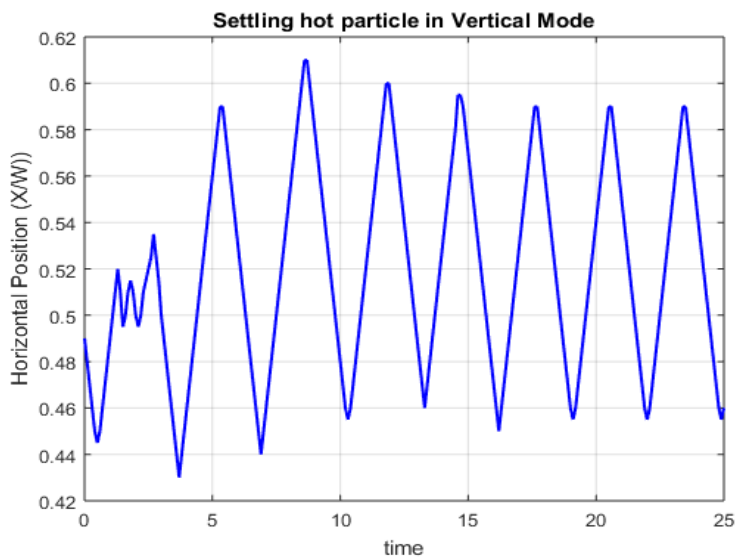

Fig.12. Horizontal position of settling hot particle w.r.t time in vertical mode

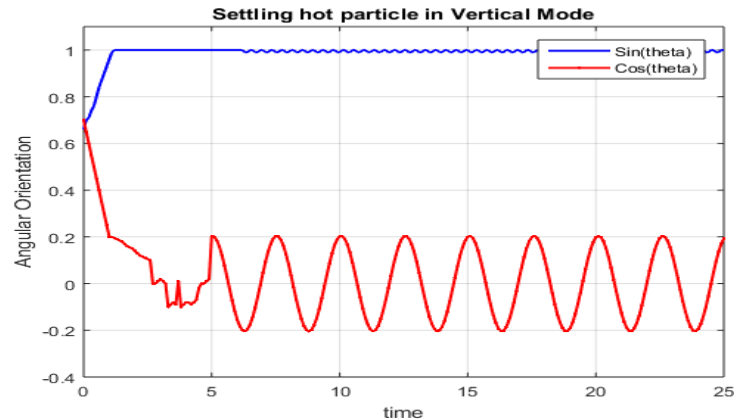

Fig.13. Angular orientation of settling hot particle w.r.t time in vertical mode

Figure 12 demonstrate that particle indicates motions of a little extent about the inside line while settling in vertical mode. Figure 13 demonstrates that the particle residue by keeping up its upstanding direction with the end goal that the point between the real hub of the oval and the horizontal course is $\mathrm{pi} / 2$.

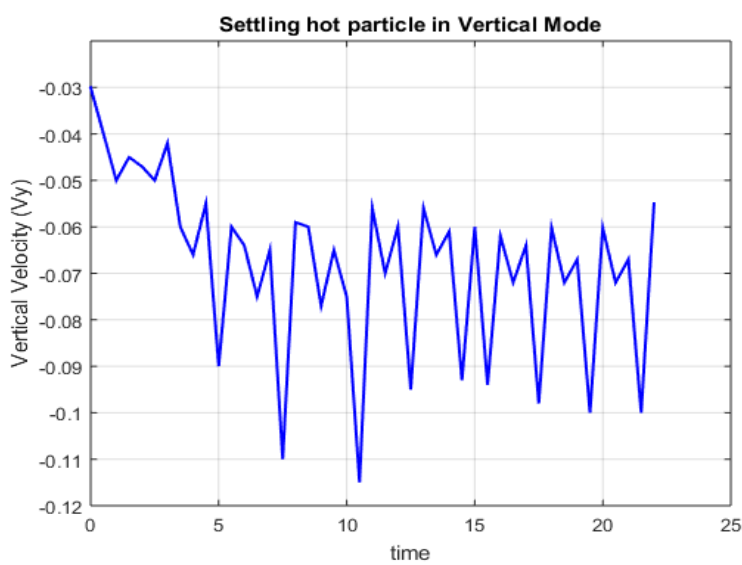

Fig.14. Vertical velocity of settling hot particle w.r.t time in vertical mode 


\section{Efficient FEM-FBM for Simulation of Elliptic Particle Sedimentation with Thermal Convection}

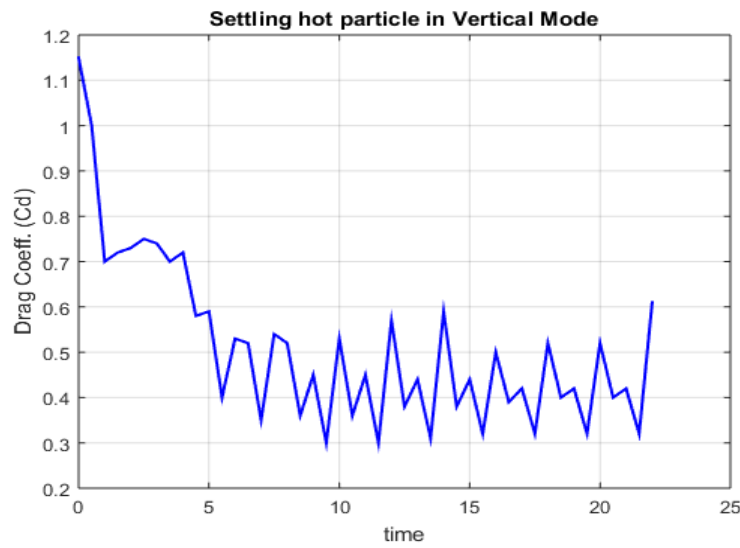

Fig.15. Drag coefficient of settling hot particle w.r.t time in vertical mode

In figure 14, the motions in the settling velocity of particle have been consequence of test among normal convection and compelled convection as discussed previously. In figure 15, drag coefficient Compact disc following up on characterised in inclined mode demonstrates comparable motions because of the changing settling velocity.

\section{B. Rolling Mode}

After the simulation of vertical mode, we further investigated the rolling mode results using the $p_{r}=1.07$ and $G r=600$. Figure 16 and 17 demonstrated the outcomes of direction and precise directions of the hot particle in rolling mode. From the results, it is seen that the particle settled from centre line close to one side divider along motions of little degree \& edge between genuine centre point of particle \& flat course modifications discontinuously from 0 to 2 pi.

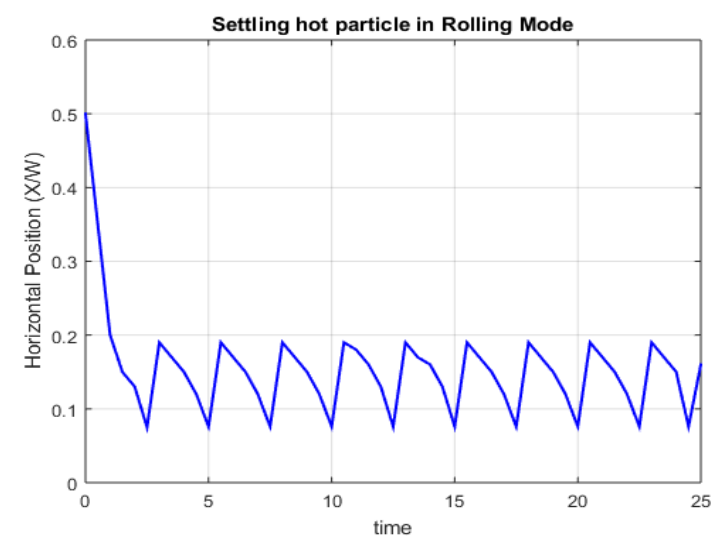

Fig.16. Horizontal position of settling hot particle w.r.t time in rolling mode

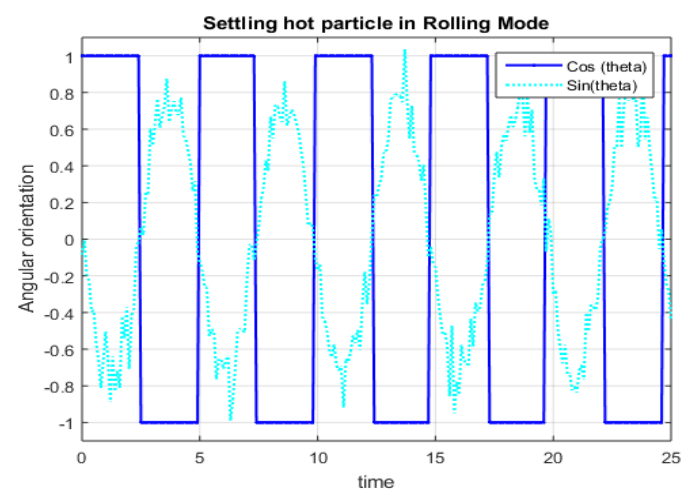

Fig.17. Angular orientation of settling hot particle w.r.t time in rolling mode

\section{Horizontal Mode}

Finally the horizontal mode case where we increased the density ratio $p_{r}=1.7$ with same $G r$ number as used in rolling mode.

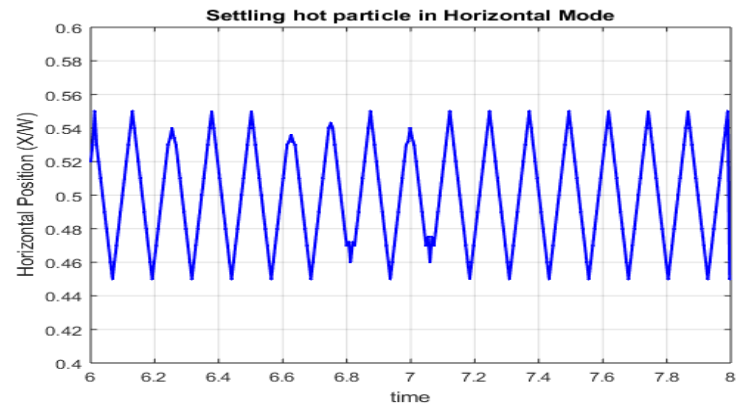

Fig.18. Horizontal position of settling hot particle w.r.t time in horizontal mode

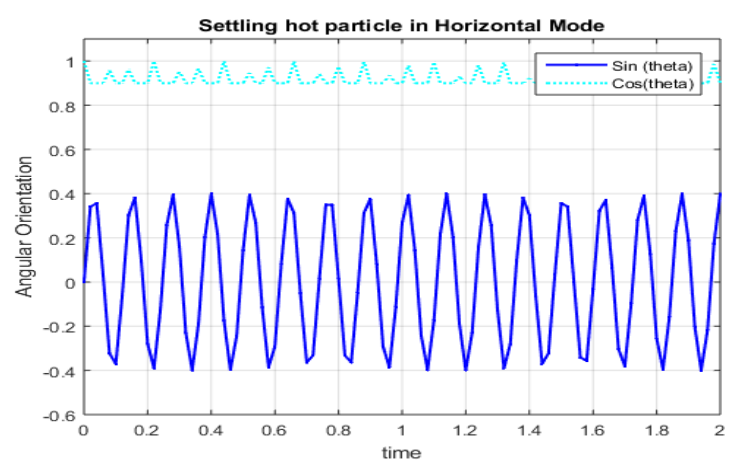

Fig.19. Angular orientation of settling hot particle w.r.t time in horizontal mode

From the analysis of all three modes, it is seen that by expanding the estimation of $\mathrm{pr}$ the particle direction and direction changing from vertical to rolling mode \& next to horizontal mode. Figures 18 and 19 are showing the outcomes of trajectories and angular orientation hot elliptical particle in horizontal settling mode respectively. Among all the modes investigation of trajectory and orientations, the variations observed for the hot elliptical particle.

\section{VALIDATION}

The validation study presented to verify the effectiveness of proposed PFEM-FBM as compared to recent method FEM-FBM [42]. The validation is performed through the comparative analysis of simulated test cases in terms of maximum and minimum values to claim the effective implementation of energy balance equation as well as to claim effectiveness of proposed model.

5.1 Validation of Different Elliptical Particle Sedimentation

In this case study we have simulated and investigated the settling behaviour of hot, cold, and isothermal elliptical particles. In this section we validated their outcomes for drag coefficient, torque velocity, and angular velocity in tables 6 , 7 and 8 respectively. As table 6 it shows that for hot settling particle its takes higher drag coefficient velocity and for isothermal it's minimum. Using the PFEM-FBM, the outcomes of drag coefficient velocity shows the more 
efficiency as compared to FEM-FBM as the PSO introduced to select the optimum solution in grid solver algorithm.

Table 6: Validation of drag coefficient for hot, cold, and isothermal particles

\begin{tabular}{|c|c|c|c|c|}
\hline Gr no & $\begin{array}{c}\text { Max } \\
\text { [FEM-FB } \\
\text { M] }\end{array}$ & $\begin{array}{l}\text { Min } \\
\text { [FEM-FB } \\
\text { M] }\end{array}$ & $\begin{array}{l}\text { Max } \\
\text { [PFEM-FB } \\
\text { M] }\end{array}$ & $\begin{array}{l}\text { Min } \\
\text { [PFEM-FB } \\
\text { M] }\end{array}$ \\
\hline $\begin{array}{c}500 \\
\text { (Hot) }\end{array}$ & 25 & 14.04 & 25 & 14 \\
\hline $\begin{array}{c}-500(\mathrm{C} \\
\text { old) }\end{array}$ & 25 & 7.6 & 25 & 7.5 \\
\hline $\begin{array}{c}0 \\
\text { (Isotherma } \\
\text { l) }\end{array}$ & 25 & 6.04 & 25 & 6 \\
\hline
\end{tabular}

Table 7: Validation of torque for hot, cold, and isothermal particles

\begin{tabular}{|c|c|c|c|c|}
\hline Gr no & $\begin{array}{l}\quad \text { Max } \\
\text { [FEM-F } \\
\text { BM] }\end{array}$ & $\begin{array}{l}\quad \text { Min } \\
\text { [FEM-F } \\
\text { BM] }\end{array}$ & \begin{tabular}{l}
\multicolumn{1}{c}{ Max } \\
[PFEM-F \\
BM]
\end{tabular} & \begin{tabular}{l}
\multicolumn{1}{c}{ Min } \\
[PFEM-F \\
BM]
\end{tabular} \\
\hline $\begin{array}{r}500 \\
\text { (Hot) }\end{array}$ & 0 & 0 & 0 & 0 \\
\hline $\begin{array}{l}-500( \\
\text { Cold) }\end{array}$ & 0.573 & $3^{-0.550}$ & 0.5662 & -0.5608 \\
\hline \begin{tabular}{l}
\multicolumn{1}{c}{0} \\
(Isother \\
mal)
\end{tabular} & $9 \begin{array}{ll}0.056 \\
9\end{array}$ & $5^{-0.054}$ & 0.0566 & -0.0561 \\
\hline
\end{tabular}

Table 8: Validation of angular velocity for hot, cold, and isothermal particles

\begin{tabular}{|c|c|c|c|c|}
\hline Gr no & $\begin{array}{c}\text { Max } \\
\text { [FEM-F } \\
\text { BM] }\end{array}$ & $\begin{array}{c}\text { Min } \\
\text { [FEM-FB } \\
\text { M] }\end{array}$ & $\begin{array}{c}\text { Max } \\
\text { [PFEM-FB } \\
\text { M] }\end{array}$ & $\begin{array}{c}\text { Min } \\
\text { [PFEM-FB } \\
\text { M] }\end{array}$ \\
\hline $\begin{array}{c}500 \\
\text { (Hot) }\end{array}$ & 0 & 0 & 0 & 0 \\
\hline-500 (Cold) & $\begin{array}{c}0.301 \\
2\end{array}$ & -0.2021 & 0.3002 & -0.2023 \\
\hline $\begin{array}{c}\text { (Isothermal } \\
\text { ) }\end{array}$ & $\begin{array}{c}0.030 \\
9\end{array}$ & -0.0200 & 0.0300 & -0.0202 \\
\hline
\end{tabular}

\subsection{Validation of Cold Elliptical Particle Settling}

The third case study belongs to study of sedimentation of cold elliptic particle has been hot fluid. For validation purpose, we validated the drag coefficient values as compared using different Grashof numbers in table 9.

Table 9: Validation of drag coefficients of settling cold particle in hot fluid

\begin{tabular}{|c|c|c|c|c|}
\hline $\begin{array}{c}\text { Gr } \\
\text { no }\end{array}$ & $\begin{array}{c}\text { Max } \\
\text { [FEM-FBM] }\end{array}$ & $\begin{array}{c}\text { Min } \\
\text { [FEM-FBM] }\end{array}$ & $\begin{array}{c}\text { Max } \\
\text { [PFEM-FBM] }\end{array}$ & $\begin{array}{c}\text { Min } \\
\text { [PFEM-FBM] }\end{array}$ \\
\hline $\begin{array}{c}-2 \\
00\end{array}$ & 0.0506 & -0.0472 & 0.0543 & -0.0243 \\
\hline $\begin{array}{c}-6 \\
000\end{array}$ & 0.0893 & -0.0868 & 0.0785 & -0.0685 \\
\hline
\end{tabular}

Table 10: Validation of settling velocity of the settling cold particle in the hot fluid

\begin{tabular}{|c|c|c|c|c|}
\hline no & $\begin{array}{c}\text { Max } \\
\text { [FEM-FBM] }\end{array}$ & $\begin{array}{c}\text { Min } \\
\text { [FEM-FBM] }\end{array}$ & $\begin{array}{c}\text { Max } \\
\text { [PFEM-FBM] }\end{array}$ & $\begin{array}{l}\text { Min } \\
\text { [PFEM-FBM] }\end{array}$ \\
\hline $0^{-20}$ & 0 & -0.39 & 0 & -0.4 \\
\hline $\begin{array}{ll} & -60 \\
0 & \\
\end{array}$ & 0 & -0.88 & 0 & -0.9 \\
\hline $00^{-10}$ & 0 & -0.73 & 0 & -0.75 \\
\hline
\end{tabular}

The validation outcomes justifies that PFEM-FBM is solid for the thermal convection recreation in particulate flows with the circularly formed particles as compared to FEM-FBM.

\section{CONCLUSION AND FUTURE WORK}

The PFEM-FBM introduced over the recent FEM-FBM method so as to improve productivity by presenting the PSO for the best arrangement determination. The re-enactment results exhibited for the diverse experiments of examinations in this work. In the primary examination, the relative investigation regards heating, cold \& isothermal elliptic particle settling exhibited which presume that torque and hydrodynamic powers following up on cold, hot \& isothermal elliptic particles demonstrates the various practices. In second and third contextual investigations, cold elliptic particle and hot elliptic particle settling into the big channels explored under the diverse settling modes. As indicated by the conduct of particle settling, distinctive settling strategies for the cold and hot particle investigated in this work, for instance, inclined mode, tumbling mode, a vertical mode, rolling more \& horizontal mode. Endorsement results legitimize that PFEM-FBM is strong for the thermal convection diversion into particulate flows along circularly shaped particles. For future work we suggest to investigate the various optimization methods in place of PSO for DSN methods.

\section{REFERENCES}

1. G. Lai, M.-C. \& Peskin, C. S. 2000 an immersed boundary method with formal second-order accuracy and reduced numerical viscosity. J. Computer. Phys. 160, 705-719.

2. Mittal, R. \& Iaccarino, G. 2005 Immersed boundary methods. Annul. Rev. Fluid Mech. 37, 239-261.

3. S. Haeri, J.S. Shrimpton, A new implicit fictitious domain method for the simulation of flow in complex geometries with heat transfer, J. Computer. Phys. 237 (2013) 21-45.

4. C. Dan, A. Wachs, Direct numerical simulation of particulate flow with heat transfer, Int. J. Heat Fluid Flow 31 (6) (2010) 1050-1057.

5. Z.G. Feng, E.E. Michael ides, Proteus: a direct forcing method in the simulations of particulate flows, J. Computer. Phys. 202 (1) (2005) 20-51.

6. X.D. Niu, C. Shu, Y.T. Chew, Y. Peng, A momentum exchange-based immersed boundary-lattice Boltzmann method for simulating incompressible viscous flows, Phys. Lett. A 354 (3) (2006) 173-182.

7. H. Zhang, Y. Tan, S. Shu, X. Niu, F.X. Trias, D. Yang, H. Li, Y. Sheng, Numerical investigation on the role of discrete element method in combined LBM-IBM-DEM modelling, Computer. Fluids 94 (2014) 37-48.

8. T.I. Zohdi, An introduction to modelling and simulation of particulate flows, SIAM, 2007.

9. C.S. Peskin, Numerical analysis of blood flow in the heart, J. Computer Phys. 25 (3) (1977) 220-252.

10. R. Glowinski, Finite element methods for incompressible viscous flow, in: Handbook of Numerical Analysis, vol. 9, 2003, pp. 3-1176.

11. S. Turek et al., the Fictitious Boundary Method for the implicit treatment of Dirichlet boundary conditions with applications to incompressible flow simulations, in: Challenges in Scientific Computing-CISC 2002, Springer, 2003, pp. 37-68.

12. D. Wan, S. Turek, Direct numerical simulation of particulate flow via multigrain FEM techniques and the fictitious boundary method, Int. J. Numeric. Meth. Fluids 51 (5) (2006) 531-566.

13. D. Wan et al., An efficient multigrain FEM solution technique for incompressible flow with moving rigid bodies, in: Numerical Mathematics and Advanced Applications, Springer, 2004, pp. 844-853. 


\section{Efficient FEM-FBM for Simulation of Elliptic Particle Sedimentation with Thermal Convection}

14. D. Wan, S. Turek, An efficient multigrain-FEM method for the simulation of solid-liquid two phase flows, J. Computer. Appl. Math. 203 (2) (2007) 561-580.

15. Z.-G. Feng, E.E. Michael ides, the immersed boundary-lattice Boltzmann method for solving fluid-particles interaction problems, J. Computer. Phys. 195 (2) (2004) 602-628.

16. R.M. MacMECCAN et al., Simulating deformable particle suspensions using a coupled lattice-Boltzmann and finite-element method, J. Fluid Mech. 618 (2009) 13-39.

17. Y. Feng et al., Combined three-dimensional lattice Boltzmann method and discrete element method for modelling fluid-particle interactions with experimental assessment, Int. J. Numerical Methods Eng. 81 (2) (2010) 229-245.

18. A. Hager et al., Parallel open source CFD-DEM for resolved particle-fluid interaction, J. Energy Power Eng. 7 (9) (2013) 1705.

19. R. Sun, H. Xiao, SediFoam: a general-purpose, open-source CFD-DEM solver for particle-laden flow with emphasis on sediment transport, Computer. Geosci. 89 (2016) 207-219.

20. J. Kim, H. Choi, An immersed boundary finite-volume method for simulations of the heat transfer in complex geometries, Korean Soc. Mech. Eng. Int. J. 18 (2004) 1026-1035.

21. J. Pacheco et al., Numerical simulations of heat transfer and fluid flow problems using an immersed-boundary finite-volume method on no staggered grids, Numerical Heat Transf., Part B: Fundam. 48 (1) (2005) $1-24$.

22. I. Demirdz 'ic' et al., Fluid flow and heat transfer test problems for non-orthogonal grids: bench-mark solutions, Int. J. Numeric. Meth. Fluids 15 (3) (1992) 329-354.

23. H. Gan et al., Direct numerical simulation of the sedimentation of solid particles with thermal convection, J. Fluid Mech. 481 (2003) 385-411.

24. H. Gan et al., Simulation of the sedimentation of melting solid particles, Int. J. Multi-ph. Flow 29 (5) (2003) 751-769.

25. Z. Yu et al., A fictitious domain method for particulate flows with heat transfer, J. Computer. Phys. 217 (2) (2006) 424-452.

26. Z.-G. Feng, E.E. Michael ides, Inclusion of heat transfer computations for particle laden flows, Phys. Fluids 20 (4) (2008) 040604.

27. Z.G. Feng, S.G. Musong, Direct numerical simulation of heat and mass transfer of spheres in a fluidized bed, Powder Technol. 262 (2014) 62-70.

28. S. Haeri, J.S. Shrimpton, A new implicit fictitious domain method for the simulation of flow in complex geometries with heat transfer, J. Computer. Phys. 237 (2013) 21-45.

29. C. Dan, A. Wachs, Direct numerical simulation of particulate flow with heat transfer, Int. J. Heat Fluid Flow 31 (6) (2010) 1050-1057.

30. J. Hu, Z. Guo, A numerical study on the migration of a neutrally buoyant particle in a Poiseuille flow with thermal convection, Int. J. Heat Mass Transf. 108 (2017) 2158-2168.

31. K. Agrawal, W. Holloway, C. C. Milioli, F. E. Milioli, and S. Sundaresan, Filtered models for scalar transport in gas-particle flows, Chem. Eng. Sci. 95, 291-300 (2013).

32. S. M. El-Behery, W. El-Askary, M. H. Hamed, and K. Ibrahim, Numerical and experimental studies of heat transfer in particle-laden gas flows through a vertical riser, Int. J. Heat Fluid Flow 33(1), 118-130 (2012).

33. J. G. Kuerten, C. Van der Geld, and B. J. Geurts, Turbulence modification and heat transfer enhancement by inertial particles in turbulent channel flow, Phys. Fluids 23(12), 123301 (2011).

34. A. Morris, Z. Ma, S. Pannala, and C. Hrenya, Simulations of heat transfer to solid particles flowing through an array of heated tubes, Sol. Energy 130, 101-115 (2016).

35. A. Frankel, H. Pouransari, F. Coletti, and A. Mani, Settling of heated particles in homogeneous turbulence, J. Fluid Mech. 792, 869-893 (2016).

36. Z. Wang, J. Fan, K. Luo, and K. Cen, Immersed boundary method for the simulation of flows with heat transfer, Int. J. Heat Mass Transfer 52(19-20), 4510-4518 (2009).

37. S. Tenneti, B. Sun, R. Garg, and S. Subramaniam, Role of fluid heating in dense gas-solid flow as revealed by particle-resolved direct numerical simulation, Int. J. Heat Mass Transfer 58(1-2), 471-479 (2013)

38. B. Sun, S. Tenneti, and S. Subramaniam, Modelling average gas-solid heat transfer using particle-resolved direct numerical simulation, Int. J. Heat Mass Transfer 86, 898-913 (2015).

39. B. Sun, S. Tenneti, S. Subramaniam, and D. L. Koch, Pseudo-turbulent heat flux and average gas-phase conduction during gas-solid heat transfer: Flow past random fixed particle assemblies, J. Fluid Mech. 798, 299-349 (2016)

40. K. Walayat, Z. Wang, K. Usman, and M. Liu, An efficient multi-grid finite element fictitious boundary method for particulate flows with thermal convection, Int. J. Heat Mass Transfer 126, 452-465 (2018).
41. A. Xu, L. Shi, and T. Zhao, Thermal effects on the sedimentation behaviour of elliptical particles, Int. J. Heat Mass Transfer 126, 753-764 (2018).

42. Khuram Walayat, Zhilang Zhang, Kamran Usman, Jianzhong Chang, and Moubin Liu, Dynamics of elliptic particle sedimentation with thermal convection, Physics of Fluids 30, 103301 (2018). 